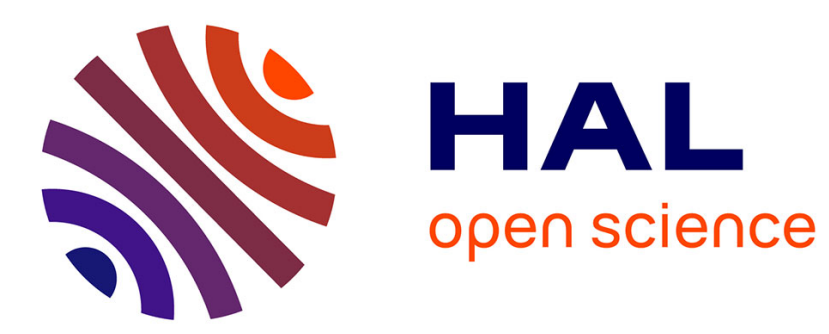

\title{
A Method for Modeling Analytical Stiffness of a Lower Mobility Parallel Manipulator
}

Olivier Company, François Pierrot, Jean-Christophe Fauroux

\section{To cite this version:}

Olivier Company, François Pierrot, Jean-Christophe Fauroux. A Method for Modeling Analytical Stiffness of a Lower Mobility Parallel Manipulator. ICRA: International Conference on Robotics and Automation, 2005, Barcelona, Spain. lirmm-00105965

\section{HAL Id: lirmm-00105965 https://hal-lirmm.ccsd.cnrs.fr/lirmm-00105965}

Submitted on 6 Jul 2016

HAL is a multi-disciplinary open access archive for the deposit and dissemination of scientific research documents, whether they are published or not. The documents may come from teaching and research institutions in France or abroad, or from public or private research centers.
L'archive ouverte pluridisciplinaire HAL, est destinée au dépôt et à la diffusion de documents scientifiques de niveau recherche, publiés ou non, émanant des établissements d'enseignement et de recherche français ou étrangers, des laboratoires publics ou privés. 


\section{A Method for Modeling Analytical Stiffness of a Lower Mobility Parallel Manipulator}

\author{
Olivier Company and François Pierrot \\ LIRMM \\ CNRS - University of Montpellier II \\ 161 rue Ada, 34392 Montpellier Cedex 5, France \\ $<$ company, pierrot $>@$ lirmm.fr
}

\author{
Jean Christophe Fauroux \\ LARAMA \\ IFMA \\ Campus des Cézeaux, BP 265, 63175 Aubière Cedex, France \\ Jean-Christophe.Fauroux@ifma.fr
}

\begin{abstract}
The $\mathbf{H} 4$ robot is a parallel machine with four degrees of freedom. The purpose of this work is to evaluate the $\mathrm{H} 4$ stiffness, $i e$ the displacement response of the tool controlled point when it is submitted to a given force using an analytical method. A stiffness analysis based on analytical calculations is performed. It has the advantage to be rather fast and easy to integrate into a design optimization. This method allows to compute stiffness matrix of parallel robots and takes into account particularity of parallel robots with articulated traveling plate. Some numerical results are shown at the end of this paper for the $\mathrm{H4}$ first prototype.
\end{abstract}

Index Terms-Parallel manipulator, Stiffness modeling, Lower mobility, Articulated traveling plate

\section{INTRODUCTION}

Parallel manipulators are still a broad topic of research and the possible mechanical architectures are not all discovered yet. Since the first publications on this topic, the major part of research efforts was devoted to six-degree of freedom (dof) manipulators [1], mainly hexapods like Gough [2] or Stewart [3] platforms.

Clavel contributes to a new phase in research on parallel manipulators by putting the focus on lower mobility parallel mechanisms by publishing his results on the Delta Architecture [4] at the end of the 80's. This four-dof robot has the same motions (Schoenflies motions [5]) as a serial SCARA arm while being light weight. As a consequence, $\mathrm{H} 4$ robot is well suited to high speed pick-and-place tasks.

A lot of authors work on improving lower manipulability parallel manipulators by finding new architectures [6]. The H4 robot created by the authors in 1999 has the same motions $i e$ three translations plus one rotation about a given axis (vertical axis has been chosen for the prototype).

Stiffness is a crucially important performance specification of parallel kinematic machines. In order to get a real industrial machine, the $\mathrm{H} 4$ prototype (Fig. 1) must be optimized. Because of its long arms and rods, designers must be particularly careful with the machine stiffness, which has direct consequences on manipulation accuracy [7]. Several studies were performed by inventors to determine the geometric model, the usable workspace and the forces into the machine components [8]. The work presented in this paper is about stiffness modeling of $\mathrm{H} 4$ robot and can be easily extended to lower manipulability parallel manipulators and manipulators with articulated traveling plate. This paper is divided in four parts:

- firstly the robot kinematics and its parameters are described

- the method is introduced

- and developed in the fourth section

- numerical results and comments are given in the last section

The conclusion of this paper mentions the feed-back of this work on the design of future parallel manipulators.

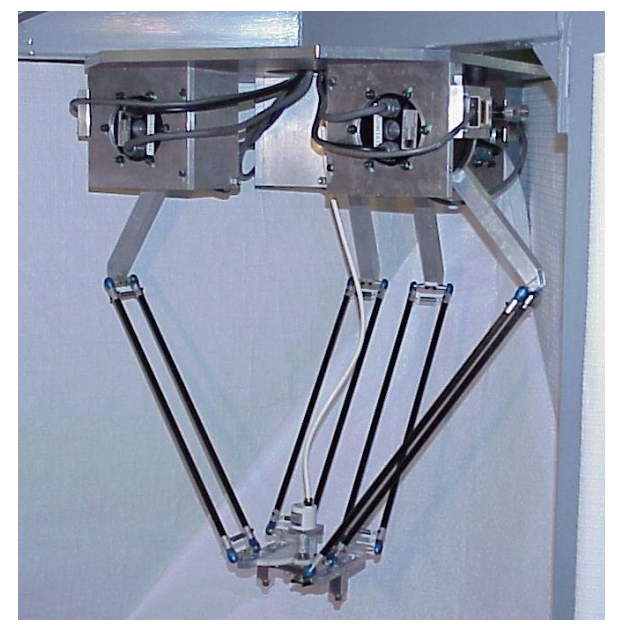

Fig. 1. H4 robot prototype

\section{ROBOT KINEMATICS DESCRIPTION}

Robot kinematics can be described by its joint-andloop graph (Fig. 2) where each box stands for a revolute (R) or spherical (S) joint (see table I). Grey boxes stand for actuated joints. Robot practical design is extremely simple thanks to the use of DD motors. Rods are made of carbon fiber; arms, forearms and traveling plate are made of aluminum alloy. Before going further robot's geometry must be modeled. As depicted on Fig. 3:

- $P_{i}$ is a point belonging to $i^{\text {th }}$ actuator's revolute axis $(i=1 . .4)$

- $\boldsymbol{u}_{\boldsymbol{i}}$ is the unitary vector defining the rotation axis

- $A_{i j}$ is the center of ball joint number $j$ on the actuator side $(j=1 . .2)$ 
- $A_{i}$ is the middle of $A_{i 1}$ and $A_{i 2}$

- $B_{i j}$ is the center of ball joint number $j$ on the traveling plate side

- $B_{i}$ is the middle of $B_{i 1}$ and $B_{i 2}$

- $v_{\boldsymbol{k}}$ is unitary vector of revolute joint on side number $k$ of the traveling plate $(k=1 . .2)$, see Fig. 4

- $C_{k}$ is the center of the revolute joint on the side number $k$ of the traveling plate

- $D$ is the end point of the robot

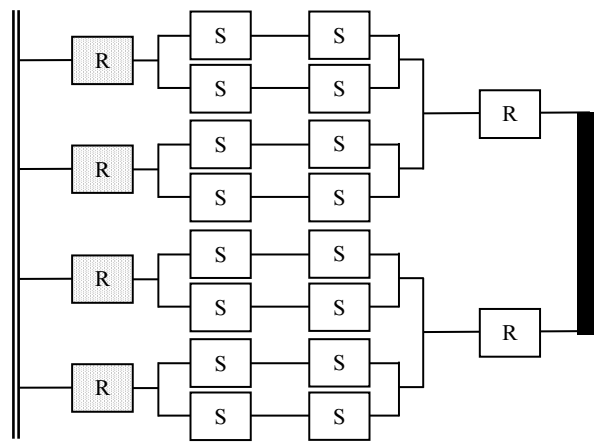

Fig. 2. H4 robot - joint-and-loop graph

TABLE I

KINEMATIC CHAINS DESCRIPTION

\begin{tabular}{|c|c||c|c|}
\hline symbol & joint name & symbol & joint name \\
\hline$P$ & prismatic & $S$ & spherical \\
\hline$R$ & revolute & $\frac{X}{\boldsymbol{X}}$ & with sensor \\
\hline$U$ & universal & actuated \\
\hline$C$ & cylindrical & & \\
\hline
\end{tabular}

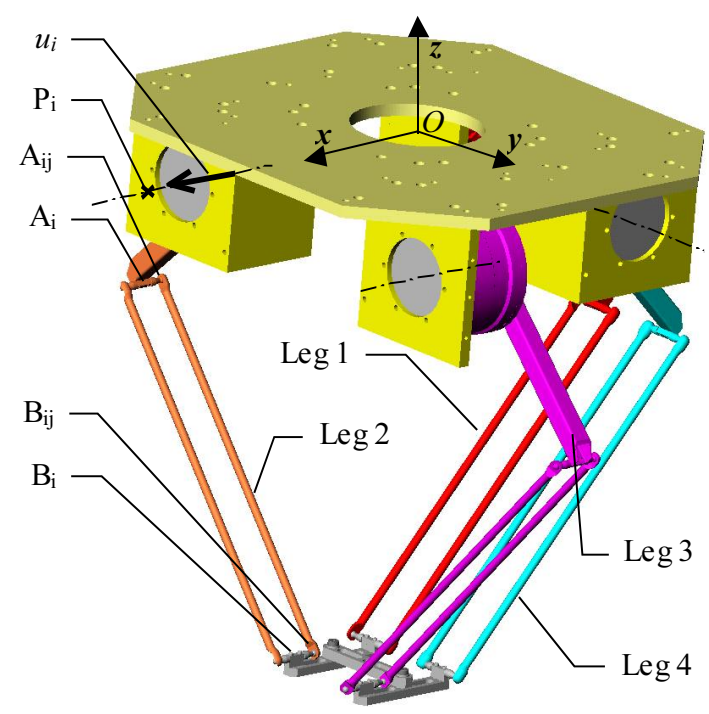

Fig. 3. H4 robot - CAD model and parameters

H4 robot prototype was built according to the following simplifying hypothesis:

- Points $P_{i}$ and $A_{i}$ are supposed to be located in the same plane perpendicular to $\boldsymbol{u}_{\boldsymbol{i}}$
- All forearms are supposed to have the same length $r$

- All bars are supposed to have the same length $l$

Geometrical constraints (these constraints are required to have robot's end point desired displacements $i e$ three translations and one rotation about $\boldsymbol{z}$ axis [9]):

- Vector defined by $A_{i 1}$ and $A_{i 2}$ is collinear to $\boldsymbol{u}_{\boldsymbol{i}}$

- Vector defined by $B_{i 1}$ and $B_{i 2}$ are collinear to $\boldsymbol{u}_{\boldsymbol{i}}$

- $v_{1}$ and $v_{2}$ are collinear to $z$ axis and are replaced by $z$ in following developments

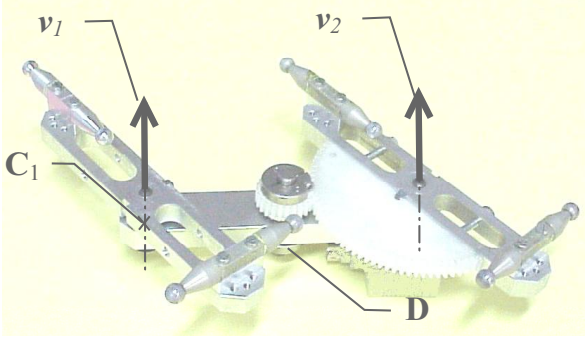

Fig. 4. H4 robot: Close view of traveling plate and parameters

As this robot has a particular architecture, some explanations about its behavior are now given. The robot is composed by four "legs" linking the fixed part (with the reference frame visible in Fig. 3) to the traveling plate. Mainly, technology and working principle is identical to Delta robot. The major improvement is based on the use of an articulated traveling plate instead of a rigid one. The shape of the traveling plate looks like letter " $\mathrm{H}$ " composed by two lateral bars linked to the central bar by two revolute joints. Each leg is composed by a forearm and a "spatial parallelogram" ie a four bar linkage with spherical joints, each side having the same length as the opposite one.

When the robot is assembled, lateral bars are parallel to each other. In such a configuration, if geometrical design rules are respected during the design stage, "spatial parallelograms" are planar. When the robot moves, excepted for singular configurations, the lateral bars remain parallel and "spatial parallelograms" planar. The end effector is linked to the central bar. Its possible displacements are three translations and one rotation about a given axis. This rotation is obtained by internal deformation of the articulated traveling plate.

When robot components don't have their theoretical geometry due to bad manufacturing process or deformation linked to external forces applied to the robot, following changes occur:

- Traveling plate doesn't remain parallel when moving

- Lateral bars don't remain parallel to each other

To extend the range of motion of the traveling plate (limited to 45 degrees) and to reach a 180-degree rotation capability in both directions, traveling plate design has been improved and equipped with a mechanical amplification system with a ratio of $4: 1$ that can be seen on Fig. 4. This device was excluded from the stiffness modeling presented in this paper. 


\section{STIFFNESS MODELING METHOD}

In this section, a method giving a good estimate of robot stiffness is presented. This method is similar to the one presented in [7] for a Gough Stewart Platform, assuming that machine elements are considered as springs. The advantage of this method is to provide results with low computation time that can easily be integrated during the robot design stage in an iterative design process or in a stiffness optimization procedure. This method is based on classical mechanical tools and equations. The application of this method to lower mobility parallel mechanisms is more difficult than the one for hexapods (presented in [7]). Moreover, $\mathrm{H} 4$ robot has particularities that must be integrated to the model and complicate this modeling:

- legs stress is not only tension/compression but also includes bending of the arms

- the traveling plate is not "rigid" because of the two passive joint added

For this method (see Fig. 5) virtual forces and torques are applied on the end point. The load of all the robot elements is derived. Giving this load, elements deformation is computed. Using small displacement theory (first order approximation), end point displacement is computed. As a final result a relation between an applied force and a resulting displacement is obtained in a linear form. Matrix contained in this relation is then considered as the robot stiffness matrix.

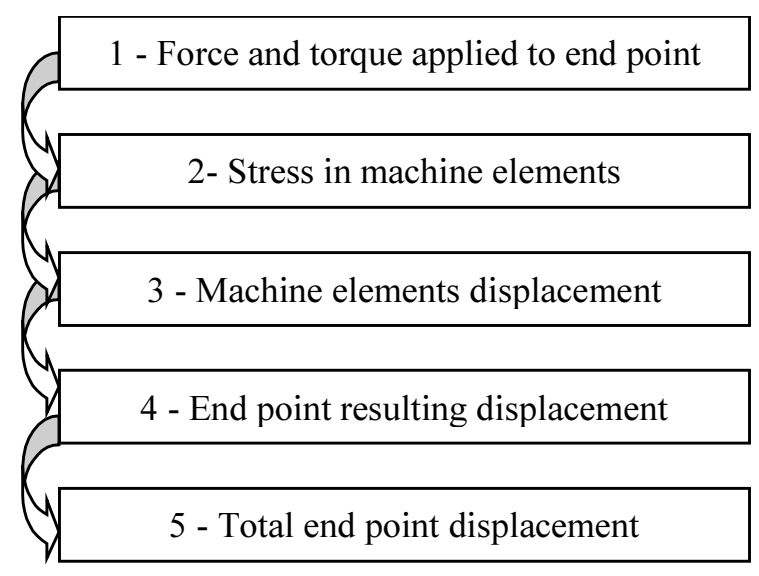

Fig. 5. Method for analytical stiffness modeling

Only a restrained set of elements are taken into account in the following derivations. The considered elements are the ones who have the lower stiffness regarding their shape and material. Stresses taken into account to compute stiffness are: actuators stiffness (control stiffness), bending in forearms, torsion in forearms and tension-compression in bars

Elements neglected are: machine frame, joints, lateral and central bars of the traveling plate and compression in forearms.

\section{Analytical DeVElopments}

\section{A. Preliminary statements}

The goal of this first subsection is to find linear relations between displacement of characteristic points and end point displacement (step 4 of the method Fig. 5). To do so, finite displacement theory is used. The following relations give machine geometrical models:

$$
\left\{\begin{array}{l}
\left\|\boldsymbol{w}_{\boldsymbol{i j}}\right\|^{2}=l_{i j}^{2} \\
i=1 . .4, j=1 . .2
\end{array}\right.
$$

where $\boldsymbol{w}_{i j}$ is the vector linking point $A_{i j}$ to $B_{i j}$. First order approximation gives:

$$
\left(\delta \boldsymbol{b}_{\boldsymbol{i j}}-\delta \boldsymbol{a}_{\boldsymbol{i j}}\right) \cdot \boldsymbol{w}_{\boldsymbol{i j}}=l_{i j} . \delta l_{i j}
$$

where $\delta \boldsymbol{a}_{\boldsymbol{i}}$ (resp. $\delta \boldsymbol{b}_{\boldsymbol{i} \boldsymbol{j}}$ ) is the change in vector linking point $O$ to $A_{i j}$ (resp. $B_{i j}$ ).

In this relation, $\delta l_{i j}$ and $\delta \boldsymbol{a}_{i j}$ depend directly on machine elements deformation. $\delta \boldsymbol{b}_{\boldsymbol{i}}$ depends on end point displacement and traveling plate displacement. When a load is applied on machine elements, their geometry change and nominal conditions expressed in section II are no longer respected. Under load, additional displacements must be considered: central bar rotation around $\boldsymbol{y}$ and $\boldsymbol{z}$ axes, and rotation of lateral bars around $\boldsymbol{v}_{\mathbf{1}}$ or $\boldsymbol{v}_{\mathbf{2}}$ axes. In fact, the traveling plate is considered as a mechanism composed by rigid bodies. A set of eight parameters is necessary to express traveling plate possible spatial displacements (including its internal dof). The vector used to model its displacement is:

$$
\delta \boldsymbol{t} \boldsymbol{p}=\left[\begin{array}{llll}
\delta \boldsymbol{D}^{T} & \delta \boldsymbol{\Omega}^{T} & \delta \omega_{1} & \delta \omega_{2}
\end{array}\right]^{T}
$$

where:

- $\delta \boldsymbol{D}$ is vector composed by Cartesian coordinates of point $D$ displacement

- $\delta \Omega$ is vector composed by rotation angles of robot end part

- $\delta \omega_{k}$ is the rotation angle of revolute joint number $k$ ( $k=1$ for $i \in 1,2, k=2$ for $i \in 3,4$ )

$$
\begin{gathered}
\delta \boldsymbol{c}_{\boldsymbol{k}}=\delta \boldsymbol{d}+\boldsymbol{s}_{\boldsymbol{k}} \times \delta \boldsymbol{\Omega} \\
\delta \boldsymbol{b}_{\boldsymbol{i j}}=\delta \boldsymbol{c}_{\boldsymbol{k}}+\boldsymbol{t}_{\boldsymbol{i} \boldsymbol{j}} \times\left(\delta \omega_{k} \boldsymbol{z}+\delta \boldsymbol{\Omega}\right)
\end{gathered}
$$

where $\delta \boldsymbol{c}_{\boldsymbol{k}}$ (resp. $\delta \boldsymbol{d}$ ) is the change in vector linking point $O$ to $C_{k}$ (resp. $D$ ) and $s_{\boldsymbol{k}}$ (resp. $\boldsymbol{t}_{\boldsymbol{i j}}$ ) is the vector linking point $C_{k}$ to $D$ (resp. $B_{i j}$ to $C_{k}$ ). All this set of equations can be easily written in a linear form:

$$
M_{1} . \delta t p=M_{2} . \delta \epsilon
$$

where:

$$
\begin{aligned}
& M_{\mathbf{1}}=\left[\begin{array}{lll}
\boldsymbol{w}_{\boldsymbol{i j}}{ }^{T} & \left(\boldsymbol{w}_{\boldsymbol{i j}} \times\left(\boldsymbol{s}_{\boldsymbol{k}}+\boldsymbol{t}_{\boldsymbol{i j}}\right)\right)^{T} & \ldots \\
\left(\boldsymbol{w}_{\boldsymbol{i j}} \times \boldsymbol{t}_{\boldsymbol{i j}}\right) \cdot \boldsymbol{z} & 0 \\
0 & \left(\boldsymbol{w}_{\boldsymbol{i j}} \times \boldsymbol{t}_{\boldsymbol{i j}}\right) \cdot \boldsymbol{z}
\end{array}\right] \\
& \boldsymbol{M}_{\mathbf{2}}=\left[\begin{array}{lc}
\boldsymbol{s p a r}\left(\left[\boldsymbol{w}_{\boldsymbol{i j}}\right]^{T}\right) & \left.\boldsymbol{d i a g}\left(\left[l_{i j}\right]^{T}\right)\right]
\end{array}\right.
\end{aligned}
$$


$\delta \epsilon=\left[\begin{array}{llllll}\delta \boldsymbol{a}_{\mathbf{1 1}} & \cdots & \delta \boldsymbol{a}_{\mathbf{4 2}} & \delta l_{11} & \cdots & \delta l_{42}\end{array}\right]$

Note that dimension of $\delta \epsilon$ is 34. spar() operator is defined as follows:

$$
\operatorname{spar}(X)=\left[\begin{array}{cccc}
X_{1} & 0 & \cdots & 0 \\
0 & X_{2} & 0 & 0 \\
0 & 0 & \ddots & 0 \\
0 & \cdots & 0 & X_{n}
\end{array}\right]
$$

where matrix $\boldsymbol{X}$ has $n$ lines and $\boldsymbol{X}_{\boldsymbol{i}}$ is the $i^{t h}$ line of matrix $\boldsymbol{X}$.

Now, displacement of all characteristic points of the mechanism are computed for a virtual force applied to the traveling plate (steps 1,2 and 3).

\section{B. Displacement of point $A$}

1) Actuator control stiffness: The kinematics I/O law of this robot can be written as:

$$
\boldsymbol{J}_{\boldsymbol{x}} . \dot{\boldsymbol{x}}=\boldsymbol{J}_{\boldsymbol{q}} \cdot \dot{\boldsymbol{q}} \quad \text { or } \quad \dot{\boldsymbol{x}}=\boldsymbol{J} . \dot{\boldsymbol{q}}
$$

where:

- $\dot{\boldsymbol{x}}=\left[\begin{array}{llll}\dot{x} & \dot{y} & \dot{z} & \dot{\theta}\end{array}\right]^{T}$ is the vector composed by cartesian and angular velocities of end point

- $\boldsymbol{J}, \boldsymbol{J}_{\boldsymbol{x}}$ and $\boldsymbol{J}_{\boldsymbol{q}}$ are jacobian matrices

- $\dot{\boldsymbol{q}}$ is the vector composed by actuators angular velocities

Let's consider a force $\boldsymbol{F}_{\boldsymbol{d}}$ and torque $\boldsymbol{T}_{\boldsymbol{d}}$ applied to the end point $D$ about possible displacement directions of the traveling plate. If there is no friction in the robot, then input power equals output power that leads to (as this relation is valid for every set of $\dot{\boldsymbol{q}}$ and $\boldsymbol{\Gamma}_{\boldsymbol{a c t}}$ ):

$$
\Gamma_{\text {act }}=\boldsymbol{J}^{T} \cdot \boldsymbol{\Gamma}_{\boldsymbol{d}}
$$

where

- $\boldsymbol{\Gamma}_{a c t}$ is the vector composed by actuators torque

- $\boldsymbol{\Gamma}_{\boldsymbol{d}}=\left[\begin{array}{ll}\boldsymbol{F}_{\boldsymbol{d}}^{T} & \boldsymbol{T}_{\boldsymbol{d}} \cdot \boldsymbol{z}\end{array}\right]^{T}$

If $k_{c}$ is the angular control stiffness of the actuator - all actuators are supposed to be identical, the angular displacement $\boldsymbol{\Delta} \boldsymbol{q}$ of the actuators is :

$$
\boldsymbol{\Delta} \boldsymbol{q}=\boldsymbol{J}^{T} \cdot \boldsymbol{\Gamma}_{\boldsymbol{d}} / k_{c}
$$

This can be written:

$$
\delta \epsilon_{\text {cont }}=M_{3} \Delta q
$$

with:

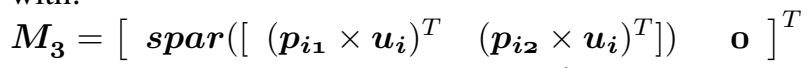$$
\text { where } \boldsymbol{p}_{\boldsymbol{i} j} \text { is the vector linking point } A_{i j} \text { to } P_{i}
$$

$$
\delta \epsilon_{\text {cont }}=M_{3} . \boldsymbol{J}^{T} . \boldsymbol{\Gamma}_{\boldsymbol{d}} / k_{c}
$$

2) Forearms bending stiffness: In this subsection, only bending moment around $\boldsymbol{u}_{\boldsymbol{i}}$ is taken into account. Bending in other directions can be negligible for the geometry of the studied robot because the biggest component of forces that produces bending is along $\boldsymbol{z}$ axis. Another reason is that the effects of bending in other directions on traveling plate displacement are lower.

Forearms are considered as constant section beams with a pure force applied to one end. This force can be expressed as:

$$
f_{f}=\Gamma_{a c t} / r
$$

Displacement of points $A_{i}$ is then perpendicular to forearms and to vector $\boldsymbol{u}_{\boldsymbol{i}}$. As all forearms shapes and material are identical, the norm of this displacement can be expressed as:

$$
\left\|\delta \boldsymbol{A}_{\boldsymbol{f}}\right\|=\frac{r^{3}}{3 . E . I} f_{f}
$$

where:

- $E$ is Young's modulus of forearm material

- $I$ is the quadratic momentum of the beam section

This leads to:

$$
\delta \epsilon_{\text {bend }}=\frac{r}{3 . E . I} \cdot \boldsymbol{M}_{\mathbf{3}} \cdot \boldsymbol{J}^{T} \cdot \boldsymbol{\Gamma}_{\boldsymbol{d}}
$$

3) Forearms torsion stiffness: Considered deformations generate a set of displacements compatible with geometrical conditions mentioned in section II. As a consequence, displacements belong to the set of possible displacements of the robot. Considered forces belong are the controlled forces ( force and torque that the robot end point can apply on an external object). The dimension of this set is four. Now, torsion deformations in forearms and tension in bars can change all the set of parameters describing traveling plate position which dimension is eight.

Let's now consider torsion effects in forearms. Firstly, forces in bars must be computed. The stress in each bar is tension-compression. Forces applied to forearms have the same direction as the considered bars. These computations need a complete set of forces and torque applied to the traveling plate applied to the traveling plate. The considered external actions are $\boldsymbol{F}_{\boldsymbol{d}}$, torque $\boldsymbol{T}_{\boldsymbol{d}}$ applied to the end point $D$ and two torques about $\boldsymbol{z}$ applied on revolute joints between central and lateral bars $t_{1}$ and $t_{2}$.

Traveling plate equilibrium leads to:

$$
\Gamma_{\text {ext }}=J_{b} F_{b}
$$

where:

- $\boldsymbol{\Gamma}_{\boldsymbol{e x t}}=\left[\begin{array}{llll}\boldsymbol{F}_{\boldsymbol{d}}{ }^{T} & \boldsymbol{T}_{\boldsymbol{d}}^{T} & t_{1} & t_{2}\end{array}\right]^{T}$ is the vector composed by extended external forces corresponding to the dual of traveling plate possible displacements expressed in eq. 8 .

- $\boldsymbol{J}_{\boldsymbol{b}}$ is the jacobian matrix relative to forces in bars

- $\boldsymbol{f}_{\boldsymbol{b}}=\left[\begin{array}{lllll}f_{b 11} & f_{b 12} & f_{b 21} & \cdots & f_{b 42}\end{array}\right]^{T}$ is the vector composed by the force in each bar ( $f_{b i j}$ is the tension load in bar number $i j$ ) 
If $\boldsymbol{J}_{\boldsymbol{b}}$ is not singular:

$$
f_{b}=J_{b}^{-1} \Gamma_{e x t}
$$

Resulting torque $\boldsymbol{t}_{\boldsymbol{t} i}$ applied on the forearm (point $A_{i}$ ) number $i$ is:

$$
\boldsymbol{t}_{\boldsymbol{t} \boldsymbol{i}}=\sum_{j} f_{i j} \boldsymbol{p}_{\boldsymbol{i} \boldsymbol{j}} \times \boldsymbol{w}_{\boldsymbol{i}}
$$

$\boldsymbol{w}_{\boldsymbol{i}}$ is an unitary vector collinear to vector linking $A_{i}$ to $B_{i}$. As for bending effects, only torsion around the forearm axis is taken into account. Torsion torque applied to forearm $i$ around its axis is:

$$
\tau_{i}=-\boldsymbol{t}_{\boldsymbol{t i}} \cdot \boldsymbol{r}_{\boldsymbol{i}}
$$

where $\boldsymbol{r}_{\boldsymbol{i}}$ is the unitary vector collinear to vector linking points $A_{i}$ and $P_{i}$

Torsion angle can be expressed as:

$$
\delta \alpha_{i}=\frac{r}{G \cdot I_{0}} \tau_{i}
$$

where $G$ is Coulomb modulus of the material and $I_{0}$ the polar momentum of the section.

Displacement of point $A_{i j}$ is:

$$
\begin{gathered}
\delta \boldsymbol{A}_{\boldsymbol{i j}}=\boldsymbol{p}_{\boldsymbol{i j}} \times \delta \alpha_{i} \boldsymbol{r}_{\boldsymbol{i}} \\
\delta \boldsymbol{A}_{\boldsymbol{i j}}=-\frac{r}{G . I_{0}}\left(\boldsymbol{p}_{\boldsymbol{i} \boldsymbol{j}} \times \boldsymbol{r}_{\boldsymbol{i}}\right) \sum_{j}\left(f_{i j} \boldsymbol{p}_{\boldsymbol{i j}} \times \boldsymbol{w}_{\boldsymbol{i}}\right) \cdot \boldsymbol{r}_{\boldsymbol{i}} \\
\delta \epsilon_{\text {tors }}=\frac{r}{G . I_{0}} \boldsymbol{M}_{\mathbf{4}} \boldsymbol{J}_{\boldsymbol{b}}^{-1} \boldsymbol{\Gamma}_{\boldsymbol{e x t}}
\end{gathered}
$$

with:

$$
\begin{aligned}
& M_{4}=\left[\begin{array}{c}
\operatorname{spar}(N) \\
\mathrm{o}
\end{array}\right] \\
& \boldsymbol{N}=\left[\begin{array}{l}
{\left[\boldsymbol{p}_{\boldsymbol{i} \mathbf{1}} \times \boldsymbol{r}_{\boldsymbol{i}}\right]^{T}\left[\boldsymbol{p}_{\boldsymbol{i} \mathbf{2}} \times \boldsymbol{r}_{\boldsymbol{i}}\right] \cdot \boldsymbol{w}_{\boldsymbol{i}}} \\
{\left[\boldsymbol{p}_{\boldsymbol{i} \mathbf{1}} \times \boldsymbol{r}_{\boldsymbol{i}}\right]^{T}\left[\boldsymbol{p}_{\boldsymbol{i} \mathbf{2}} \times \boldsymbol{r}_{\boldsymbol{i}}\right] \cdot \boldsymbol{w}_{\boldsymbol{i}}}
\end{array}\right. \\
& \left.\begin{array}{l}
{\left[\boldsymbol{p}_{\boldsymbol{i} \mathbf{2}} \times \boldsymbol{r}_{\boldsymbol{i}}\right]^{T}\left[\boldsymbol{p}_{\boldsymbol{i 1}_{\mathbf{1}}} \times \boldsymbol{r}_{\boldsymbol{i}}\right] \cdot \boldsymbol{w}_{\boldsymbol{i}}} \\
{\left[\boldsymbol{p}_{\boldsymbol{i} \mathbf{2}} \times \boldsymbol{r}_{\boldsymbol{i}}\right]^{T}\left[\boldsymbol{p}_{\boldsymbol{i}_{\mathbf{1}}} \times \boldsymbol{r}_{\boldsymbol{i}}\right] \cdot \boldsymbol{w}_{\boldsymbol{i}}}
\end{array}\right]
\end{aligned}
$$

\section{Changes in bars length}

Changes in bars length are linked to bars stiffness. According to derivations made in previous sections, forces in bars are given by the relation:

$$
\boldsymbol{F}_{\boldsymbol{b}}=\boldsymbol{J}_{\boldsymbol{b}}{ }^{-1} \boldsymbol{\Gamma}_{\boldsymbol{e x t}}
$$

Change in bars length is:

$$
\delta \boldsymbol{l}=\frac{l}{S_{b} \cdot E_{b}} \boldsymbol{f}_{\boldsymbol{b}}
$$

where $S_{b}$ is the area of bars section and $E_{b}$ is Young's modulus of bars material

$$
\delta \boldsymbol{l}=\frac{l}{S_{b} \cdot E_{b}} \boldsymbol{J}_{\boldsymbol{b}}^{-1} \boldsymbol{\Gamma}_{\boldsymbol{e x t}}
$$

$$
\delta \epsilon_{\text {tens }}=\frac{l}{S_{b} \cdot E_{b}} M_{5} J_{b}^{-1} \Gamma_{e x t}
$$

with:

$$
M_{\mathbf{5}}=\left[\begin{array}{c}
\mathbf{0} \\
\operatorname{diag}(\operatorname{ones}(1,8))
\end{array}\right]
$$

\section{Machine end point final displacement}

The following relation gives machine end point final displacement obtained when forces and torque are applied to the traveling plate (step 5) by summing all displacements:

$$
\delta \boldsymbol{t p}=M_{1}{ }^{-1} M_{\mathbf{2}}\left(\delta \epsilon_{\text {cont }}+\delta \epsilon_{\text {bend }}+\delta \epsilon_{\text {tors }}+\delta \epsilon_{\text {tens }}\right)
$$

And the following expression is derived:

$$
\delta t p=K \Gamma e x t
$$

where $\boldsymbol{K}$ is defined here as the stiffness matrix:

$$
\begin{gathered}
\boldsymbol{K}=\boldsymbol{M}_{\mathbf{1}}{ }^{-1} \boldsymbol{M}_{\mathbf{2}}\left(\frac{1}{k_{c}} M_{3} J^{T} R+\cdots\right. \\
\left.M_{3} \frac{r}{3 E . I} J^{T} R+\frac{r}{G \cdot I_{0}} M_{4} J_{b}^{-1}+\frac{l}{S_{b} \cdot E_{b}} M_{5} J_{b}^{-1}\right)
\end{gathered}
$$

with:

$$
\boldsymbol{R}=\left[\begin{array}{llllllll}
1 & 0 & 0 & 0 & 0 & 0 & 0 & 0 \\
0 & 1 & 0 & 0 & 0 & 0 & 0 & 0 \\
0 & 0 & 1 & 0 & 0 & 0 & 0 & 0 \\
0 & 0 & 0 & 0 & 0 & 1 & 0 & 0
\end{array}\right]
$$

As mentioned in the beginning of subsection IV-B.3, bending and control stiffness need a four dimension force input, torsion and tension need a eight dimension force input. As the final displacement is the sum of all these displacements for a given force, the selection matrix $\boldsymbol{R}$ must be used.

\section{STIFFness EVALUATION}

In this section, results of stiffness analytical modeling are compared to some experimental stiffness measurements on $\mathrm{H} 4$ robot prototype.

\section{A. Dimensions and mechanical characteristics}

Dimensions of the modeled prototype are (dimensions are expressed in millimeters):

$$
\begin{aligned}
& \boldsymbol{P}=\left[\begin{array}{cccc}
200 & 60 & 60 & -200 \\
-60 & -200 & 200 & 60 \\
0 & 0 & 0 & 0
\end{array}\right] \\
& \boldsymbol{U}=\left[\begin{array}{cccc}
0 & 1 & -1 & 0 \\
-1 & 0 & 0 & -1 \\
0 & 0 & 0 & 0
\end{array}\right] \\
& r=260 ; l=480
\end{aligned}
$$

$$
\boldsymbol{D B}_{\text {nominal }}=\left[\begin{array}{cccc}
-60 & 60 & 60 & -60 \\
-60 & -60 & 60 & 60 \\
50 & 50 & 50 & 50
\end{array}\right]
$$




$$
\begin{aligned}
& k_{c}=7,4.10^{6} \mathrm{Nmm}^{\circ}{ }^{\circ} \\
& E=70000 \mathrm{Nmm}^{-2} \\
& I=1,6.10^{4} \mathrm{~mm}^{4} \\
& G=0,4 E \\
& I_{0}=2 I \\
& S_{b}=28 \mathrm{~mm}^{2} \\
& E_{b}=200000 \mathrm{Nmm}^{-2}
\end{aligned}
$$

\section{B. Numerical results}

Experimental stiffness measurements were done on $\mathrm{H} 4$ robot, by applying a force on the traveling plate and measuring the resulting displacements about three directions $(\boldsymbol{x}, \boldsymbol{y}$ and $\boldsymbol{z}$ ) using dial indicators (see Fig. 6 and and a detailed description in [10]).

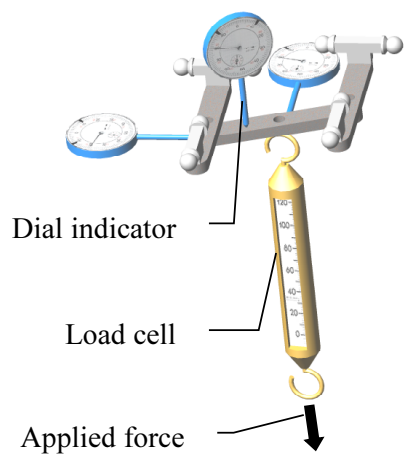

Fig. 6. Experimental setting scheme

Results obtained using these two different methods are presented in table II. Only translational response of the mechanism in $\boldsymbol{x}, \boldsymbol{y}$ and $\boldsymbol{z}$ directions is measured for forces applied in $\boldsymbol{x}, \boldsymbol{y}$ and $\boldsymbol{z}$ directions. Measurements have been done for one pose that is workspace center $i e$ all actuators angle $q_{i}$ is equal to 45 . Its is obvious that, due to its particular architecture, when a force is applied on the robot, the resulting displacement direction is not the same as the one of the force. Number given in table II are translational stiffnesses for a given pose expressed in $m / N$.

TABLE II

COMPARISON BETWEEN EXPERIMENTAL AND COMPUTED RESULTS

\begin{tabular}{|c|c|c|c||c|c|c||c|c|c|}
\hline & \multicolumn{3}{|c|}{ force along $\mathrm{x}$} & \multicolumn{3}{c|}{ force along $\mathrm{y}$} & \multicolumn{3}{|c|}{ force along $\mathrm{z}$} \\
\hline & $\mathrm{x}$ & $\mathrm{y}$ & $\mathrm{z}$ & $\mathrm{x}$ & $\mathrm{y}$ & $\mathrm{z}$ & $\mathrm{x}$ & $\mathrm{y}$ & $\mathrm{z}$ \\
\hline measured & 51 & -3 & 15 & 0 & 15 & 0 & 11 & -1 & 9 \\
\hline computed & 40 & 0 & 21 & 0 & 21 & 0 & 21 & 0 & 19 \\
\hline
\end{tabular}

The main result is that stiffness in $\boldsymbol{x}$ direction is bad regarding other directions. This stiffness study shows that the choice of robot actuators location on the machine frame does not provide good results.

The estimated results and the measured ones are "similar" due to approximations in the modeling phase. But estimated results are pessimistic regarding measured ones because some technological considerations have not been taken into account. All forearms length are considered to be stressed in bending and torsion, but in the prototype assembly, only $2 / 3$ of the forearms length are stressed.
More over, these parts are the weak point of the prototype regarding stiffness.

\section{CONCLUSION}

In this paper a method for stiffness modeling of lower mobility parallel manipulators is presented and applied to a $\mathrm{H} 4$ robot whose particularity is to have an articulated traveling plate. This analytical method is very fast and useful for the design stage. For better quality results, a FEM analysis can be conducted once all the dimensions of robot parts are chosen. Analytical results are compared to experimental results and allow to have an interesting feed-back on the design of future parallel H4-like robots:

- actuators placement (that implies legs placement) is bad and cannot be modified into a symmetrical placement because of the architecture: the robot has a null stiffness in one direction for a symmetrical placement

- as a consequence, a new articulated traveling plate (with a different kinematics) has to be designed to allow a symmetrical design of the robot. This will contribute to reduce stiffness anisotropy.

- forearms shape and material need to be modified as they are the weak point of the robot

\section{REFERENCES}

[1] J. P. Merlet, Les robots paralèles, 2nd ed., ser. robotique. Paris, France: Hermès, 1997.

[2] V. E. Gough, "Contribution to discussion of papers on research in automotive stability, control and tyre performance," in Proc. Auto. Div., Institute of mechanical engineering, 1956-1957.

[3] D. Stewart, "A platform with 6 degrees of freedom," in Proc. Inst. Mech. Ing., 1965, pp. 371-386, vol. 180, (part 1,15).

[4] R. Clavel, "Delta, a fast robot with parallel geometry," in 18th Int. Symp. on Industrial Robots. Lausanne: IFS Publications, april 1988, pp. 91-100.

[5] J. M. Hervé, "The lie group of rigid body displacements, a fundamental tool for mechanism design," Mechanism and Machine Theory, vol. 34, pp. 719-730, 1999.

[6] T. Brogardh, "PKM Research - important issues, as seen from a product development perspective at ABB robotics," in Workshop on Fundamental Issues and Future Research Directions for Parallel Mechanisms and Manipulators. Quebec City, Quebec, Canada: Clément M. Gosselin and Imme Erbert-Uphoff, editors, 2002.

[7] M. M. Svinin, F. Marquet, O. Company, and T. Gil, "On the stiffness ans stability of gough stewart platforms," in Proc. of IEEE ICRA: Int. Conf. on Robotics and Automation, Seoul, Korea, May 21-26 2001.

[8] F. Pierrot, S. Hosoe, and M. Uchiyama, "H4 parallel robot: modeling, design and preliminary experiments," in Proc. of IEEE ICRA: Int. Conf. on Robotics and Automation, Seoul, Korea, May 21-26 2001.

[9] F. Pierrot and O. Company, "H4: a new family of 4-dof parallel robots," in AIM'99: IEEE/ASME International Conference on Advanced Intelligent Mechatronics, Atlanta, Georgia, USA, september 1999, pp. 508-513.

[10] C. Corradini, J. C. Fauroux, S. Krut, and O. Company, "Evaluation of a 4-degree of freedom parallel manipulator stiffness," in Proc. 11th World Congress in Mechanism and Machine Science, IFTOMM'2003, Tianjin, China, August 18-21 2003. 